\title{
Causação cumulativa, progresso tecnológico endógeno e taxa de câmbio real em uma abordagem dinâmica de mudança estrutural *
}

\author{
Ricardo Azevedo Araujo ** \\ Matheus Silva de Paiva ${ }^{* * *}$ \\ Guilherme Jonas Costa da Silva ${ }^{* * * *}$
}

\begin{abstract}
Resumo
O trabalho tem por objetivo desenvolver um modelo multissetorial de causação cumulativa com progresso tecnológico endógeno e taxa de câmbio real. A literatura export-led growth, baseada inicialmente no modelo Dixon and Thirlwall (1979), avançou para uma abordagem multissetorial segundo Araujo (2013). Entretanto, o modelo multissetorial originalmente desenvolvido por Araujo e Lima (2007), desconsidera a taxa de câmbio e mantém a taxa de variação da produtividade do trabalho nula. Assim, pretende-se avançar no debate teórico, introduzindo no modelo Pasinettiano o progresso tecnológico endógeno e a taxa de câmbio real na Lei de Thirlwall Multissetorial. Essa contribuição torna-se importante por possibilitar aos policy makers identificar quais os setores que mais contribuem para o crescimento de longo prazo da economia doméstica, bem como propor políticas econômicas e industriais que melhorem as relações comerciais e a competitividade da economia, para conduzir a economia para um estágio superior de desenvolvimento.
\end{abstract}

Palavras-chave: Mudança estrutural; Causação cumulativa; Progresso tecnológico endógeno; Taxa de câmbio real; Crescimento econômico.

\begin{abstract}
A structural economic dynamic approach to cumulative causation, endogenous technological progress and real exchange rate

The present study aims to develop a multi-sectoral cumulative causation model with endogenous technological progress and the real exchange rate. The literature on export-led growth, originally based on the model constructed by Dixon and Thirlwall (1979), advanced to a multi-sectoral approach developed by Araujo (2013). However, the multisectoral model originally developed by Araujo and Lima (2007) disregards the exchange rate and maintains the rate of change in labor productivity at null. Thus, we intend to advance the theoretical debate by introducing endogenous technological progress and the real exchange rate in the Law of Multi-Sectoral Thirlwall to the Pasinettian model. The study makes an important contribution in terms of enabling policy makers to identify which sectors contribute most to the long-term growth of the domestic economy as well as providing economic and industrial policies to improve trade relations and the competitiveness of the economy, to drive the economy to a higher stage of development.
\end{abstract}

Keywords: Structural change; Cumulative causation; Endogenous technological progress; Real exchange rate; Economic growth.

JEL E12, F43; O41.

\footnotetext{
* Artigo recebido em 4 de fevereiro de 2015 e aprovado em 21 de maio de 2018. Os autores gostariam de agradecer o apoio financeiro do CNPq durante o desenvolvimento desta pesquisa. Evidentemente, quaisquer erros ou omissões remanescentes são de nossa inteira responsabilidade.

${ }^{* *}$ Professor do Programa de Pós-Graduação em Economia da Universidade de Brasília (UNb), Brasília, DF, Brasil. Email: rsaaraujo@unb.br.

${ }^{* * *}$ Professor do Programa de Pós-Graduação em Governança, Tecnologia e Inovação da Universidade Católica de Brasília (UCB), Brasília, DF, Brasil. E-mail: matheus.paiva@ucb.br.

${ }^{* * * *}$ Professor do Programa de Pós-Graduação em Economia da Universidade Federal de Uberlândia (UFU), Uberlândia, MG, Brasil. E-mail: guilhermejonas@ie.ufu.br.
} 
Ricardo Azevedo Araujo, Matheus Silva de Paiva, Guilherme Jonas Costa da Silva

\section{Introdução}

Embora causação cumulativa e mudança estrutural sejam conceitos até certo ponto ausentes na economia ortodoxa, eles são conceitos centrais da escola de pensamento pósKeynesiana, em que o crescimento é liderado pela demanda e não pela oferta (Ocampo, 2005; Cornwall e Cornwall, 2002; Setterfield, 1997). O conceito de causação cumulativa foi utilizado por vários autores tais como Kaldor (1966, 1967), Skott (1988), McCombie e Thirlwall (1994), para descrever um dos efeitos lógicos de economias de escala estáticas e dinâmicas, ou o que é considerado retornos crescentes num sentido mais amplo. De acordo com essa visão, as manufaturas desempenham uma função especial na geração de aumento da produtividade da economia como um todo, como consequência da existência de retornos crescentes de escala que se espalham ao longo da economia devido aos seus encadeamentos produtivos para frente e para trás.

Nesses modelos, a demanda desempenha um importante papel no crescimento econômico e um aumento da mesma, através da expansão das exportações, cria condições propícias para o aumento da oferta e, portanto, para a expansão econômica. Essa linha de argumentação foi utilizada, por exemplo, como suporte de estratégias de crescimento econômico que são conhecidas como "crescimento voltado para fora". De acordo com essa visão, a incorporação de novos mercados consumidores criaria no país exportador um ambiente propício não apenas para a expansão da produção, mas também para a absorção de progresso tecnológico (Bruton, 1998).

O problema da abordagem é que apesar de os escritos formais de Kaldor terem uma forte ênfase na questão setorial da economia, os modelos de causação cumulativa geralmente são construídos de forma agregada, ficando sujeitos então à mesma crítica do modelo de Solow. Uma forma de se contornar essa dificuldade é se construir uma ponte entre as abordagens Pasinettiana e Kaldoriana, introduzindo-se funções de Kaldor-Verdoorn setoriais. Tal abordagem permite criar um link entre a oferta e a demanda no modelo Pasinettiano e, também, permite que se conduza a análise de causação cumulativa em um esquema analítico multissetorial, onde as mudanças estruturais passam a ser endogeneizadas. Esse é um dos objetivos da abordagem de dinâmica de mudança estrutural estendida para o comércio internacional. O primeiro passo nessa ligação entre as teorias Pasinettianas e Kaldorianas, foi sem dúvida a derivação de uma versão multissetorial da Lei de Thirlwall a partir do modelo Pasinettiano (Araujo; Lima, 2007).

Apesar de o modelo Pasinettiano apresentar uma economia multissetorial, em sua versão original, o progresso tecnológico é exógeno e, desse modo, se exclui qualquer relação circular entre o motor do crescimento econômico, ou seja, o aumento da produtividade do trabalho e variações na demanda motivadas pela Lei de Engel. Isso é problemático, uma vez que o progresso tecnológico é o responsável pelo aumento da produtividade do trabalho e da renda per capita que por sua vez se traduz em aumentos da demanda que favorecem bens com elasticidade-renda da demanda maior. Na medida em que não há nenhum mecanismo de 
"feedback" desse aumento da demanda em relação à nova geração de progresso tecnológico, as mudanças estruturais permanecem exógenas, dependendo muito mais de parâmetros como taxa exógena de progresso tecnológico do que da interação cumulativa entre os lados da oferta e demanda. Numa perspectiva mais inclusiva, deve-se considerar a existência de eventuais canais de ligação entre oferta e demanda no sentido de se permitir uma endogeneização do progresso tecnológico. Na medida em que essa tarefa for cumprida, pode-se almejar uma teoria endógena de mudanças estruturais e não uma em que de antemão já se saibam quais são as causas da mudança estrutural, sejam elas ou de oferta ou de demanda.

Apesar de a abordagem Pasinettiana apresentar a clara vantagem de permitir que se atribua a cada setor uma taxa particular de aumento da produtividade, o progresso tecnológico é tratado ainda de forma exógena no seu esquema. Alguns autores tentaram endogeneizar o progresso tecnológico. Pode-se citar entre eles Reati (1998) que introduziu "long waves" para explicar revoluções tecnológicas no modelo Pasinettiano. Araujo e Teixeira (2011) e D’Agata (2010) também buscaram essa endogeneização, mas considerando uma abordagem evolucionária de "dynamic capabilities" como uma força fundamental determinando o progresso tecnológico.

Embora essas abordagens tenham se mostrado úteis, elas não levam em consideração a interação entre demanda e oferta na geração do progresso tecnológico e, nesse sentido, não são capazes de abordar as conexões entre crescimento da produtividade e do produto apontadas pela tradição Kaldoriana e enfatizadas por Roberts e Setterfield (2007). De acordo com esses autores há, na verdade, uma concepção de aumento da produtividade endógeno associada com a noção de causação cumulativa, em que a taxa de progresso tecnológico é sensível à taxa de crescimento do produto. De acordo com essa noção, "o crescimento depende do crescimento" e, adicionando uma dimensão temporal a essa relação, o crescimento em um determinado ponto do tempo depende da sua própria trajetória passada em um esquema de causação cumulativa.

Ao ignorar a causação cumulativa, a abordagem de Dinâmica de Mudança Estrutural não leva em consideração uma importante dimensão do crescimento econômico, o que reduz a qualidade de tal abordagem. Nesse sentido, um dos objetivos do terceiro tópico é endogeneizar o progresso tecnológico no modelo Pasinettiano, tornando-o sensível aos desenvolvimentos da teoria do crescimento puxado pela demanda em que a taxa de aumento da produtividade é função da taxa de crescimento da economia através da operação da Lei de Kaldor-Verdoorn. O próprio Pasinetti (1983, p.69) reconhece a importância de se considerar o lado da demanda quando se estudam as origens do progresso tecnológico. De acordo com ele,

this means that any investigation into technical progress, must necessarily imply some hypothesis on the evolution of consumers' preferences as income increases. Not to make such hypothesis and to pretend to discuss technical progress without considering the evolution of demand would make it impossible to evaluate the very relevance of technical progress and would render the investigation itself meaningless. 
Ricardo Azevedo Araujo, Matheus Silva de Paiva, Guilherme Jonas Costa da Silva

Através dessa abordagem, uma noção mais aprofundada de progresso tecnológico, como ressaltada por Roberts e Setterfield é levada em conta, fazendo com que o modelo Pasinettiano se torne consistente com a ideia de causação cumulativa. O resultado então é o surgimento de um modelo multissetorial de mudança estrutural e causação cumulativa, em que o ritmo do progresso tecnológico pode ser determinado endogenamente.

É interessante notar que alguns autores já tentaram estabelecer essa ligação entre mudança estrutural e causação cumulativa. Botta (2009), por exemplo, enfatiza essa ligação em um modelo de crescimento restrito pelo balanço de pagamentos em que a expansão do setor industrial e da economia como um todo se alimentam mutuamente, dando origem a um processo cumulativo. Contudo, essa análise enfrenta limitações pois a análise é conduzida em um esquema agregado.

Outro autor que também trata da questão é Fiorillo (2001). Esse autor simula como a taxa de crescimento e de especialização da economia evoluem no decorrer do tempo em um modelo de crescimento liderado pelas exportações num estilo Kaldoriano. O seu foco é sobre a interação entre as taxas de crescimento do produto e as elasticidades renda das exportações, levando em consideração a presença de taxas endógenas de crescimento nos regimes de acumulação. Isso significa que o padrão de especialização de um país em um determinado setor faz com que esse padrão seja reforçado no decorrer do tempo. Esse resultado é confirmado aqui tanto do ponto de vista analítico quando computacional. Uma vez que um dos países é capaz de obter vantagens comparativas em um determinado setor, a tendência é que essa vantagem seja mantida no decorrer do tempo, uma vez que ela dará origem a um processo de causação cumulativa que tende a aumentar não apenas a competividade como também a produtividade do país naquele setor específico. Essa visão está de acordo também com o que foi reportado por Krugman

Basically, once a pattern of specialization is established, it remains unchanged, with changes in relative productivity acting to further lock the pattern in. (...) Like a river which digs its own bed deeper, a pattern of specialization, once established, will induce relative productivity changes which strengthen the forces preserving that pattern (1987, p. 46-47).

Isso significa que uma estratégia de crescimento voltado para fora pode permitir ao país atrasado obter acesso a mercados consumidores com renda per capita maior do que a sua própria, permitindo assim que esses países passem a concentrar a sua estrutura produtiva na produção e exportação de bens com maior elasticidade-renda da demanda. Processos como o de "learning by doing" que surgem a partir de retornos de escala podem permitir a aquisição e a assimilação de novas técnicas de produção que podem aumentar significativamente a produtividade. Como consequência, podem surgir mudanças estruturais em que a mão-de-obra esteja migrando de setores de baixa produtividade para setores com mais alta produtividade.

Para tanto, o trabalho está estruturado em mais quatro seções, além desta introdução. Na primeira seção, apresenta-se uma breve revisão da literatura em torno da Lei de Thirlwall. Na 
Causação cumulativa, progresso tecnológico endógeno e taxa de câmbio real em uma abordagem dinâmica...

sequência, a atenção volta-se para a endogeneização do progresso tecnológico no modelo Pasinettiano e sua endogeneização pela Lei de Kaldor-Verdoorn. Na terceira seção, apresentase uma versão estendida do modelo multissetorial, com câmbio real e progresso tecnológico endógeno, bem como suas principais implicações sobre o crescimento econômico. Na última seção, algumas considerações finais são apresentadas.

\section{Dinâmica das exportações e crescimento de longo prazo: uma síntese do debate emtorno da Lei de Thirlwall (1979)}

O modelo de crescimento desenvolvido por Thirlwall (1979) tem como hipótese central tentar explicar porque existem diferentes taxas de crescimento entre os países, através de uma análise da demanda. No caso de uma economia aberta, a principal restrição da demanda é o balanço de pagamentos. De acordo com McCombie \& Thirlwall (1994), a importância de um balanço saudável de pagamentos pode ser explanada sucintamente da seguinte forma:

If a country get into balance-of-payments difficulties as it expands demand before the short-term capacity growth rate is reached, then demand must be curtailed; supply is never fully utilised; investment discouraged; technological progress is slowed down, and a country's goods compared with foreign goods become less deserable so worsening the balance of payments still further, and so on. A vicious circleis started (Mccombie; Thirlwall, 1994, p. 21).

Por outro lado, se um país é hábil ao expandir a demanda utilizando toda a capacidade produtiva existente sem surgir dificuldades no balanço de pagamentos, a pressão da demanda sobre a capacidade pode aumentar significativamente a taxa de crescimento da economia. $\mathrm{O}$ estímulo pode ser feito de várias formas:

1) Via encorajamento do investimento, que aumentaria o estoque de capitais, trazendo consigo o progresso tecnológico e o aumento da produtividade;

2) O movimento de fatores de produção dos setores com baixa produtividade para aqueles com elevada produtividade e a habilidade de importar mais pode aumentar a capacidade produtiva da economia tornando os recursos mais produtivos.

Nesse último argumento, está subentendida a teoria do crescimento conduzido pelas exportações, isso porque, é somente através da expansão das exportações, é que a taxa de

crescimento pode ser aumentada sem que o balanço de pagamentos se deteriore ao mesmo tempo.

O modelo de crescimento Thirlwall (1979), parte do pressuposto que o crescimento das exportações podem favorecer o crescimento econômico, mas países que possuem taxas de crescimento das exportações semelhantes, não necessariamente implicariam em taxas de crescimento econômico semelhantes, pois temos que observar as elasticidade-renda da demanda, devido ao fato das importações requeridas com o crescimento diferirem entre os 
Ricardo Azevedo Araujo, Matheus Silva de Paiva, Guilherme Jonas Costa da Silva

países, tendo em vista que alguns países teriam que forçar a demanda mais cedo do que outros para o equilíbrio no balanço de pagamentos.

A Lei de Thirlwall afirma que a taxa de crescimento de um país será restringida por seu desempenho nas trocas externas:

$$
y_{b}=\frac{\varepsilon \cdot z}{\pi}=\frac{x}{\pi}
$$

em que $y_{b}$ é a taxa de crescimento do produto, $x$ é a taxa de crescimento das exportações e $\pi$ é a elasticidade-renda das importações. Portanto, a taxa de crescimento com equilíbrio no balanço de pagamentos é igual à taxa de crescimento do volume das exportações dividido pela elasticidade-renda da demanda por importações.

Diversos trabalhos tentaram aperfeiçoar a "Lei de Thirlwall", principalmente, porque alguns países não se ajustaram bem à lei original. Cientes disso, Thirlwall e Hussain (1982) apresentam uma versão estendida do modelo anterior, que incorpora os fluxos de capitais, pois o endividamento fazia com que os países em desenvolvimento crescessem mais rápido que a restrição no balanço de pagamentos permitiria. A ideia é que a taxa de crescimento com restrição no balanço de pagamentos começa com um desequilíbrio em transações correntes, devido às importações, mas é financiada pela soma ponderada do crescimento das exportações com o crescimento do fluxo de capitais, dividido pela elasticidade-renda da demanda por importações. O modelo desenvolvido por Thirlwall e Hussain (1982) é sem dúvida um arcabouço teórico muito útil para a análise empírica das economias em desenvolvimento. No entanto, seu tratamento do capital estrangeiro ignora as potenciais complicações introduzidas pela acumulação do endividamento externo, que têm frequentemente desorganizado processos de crescimento de economias aparentemente saudáveis e fortes.

Portanto, apesar de considerar a entrada de capital estrangeiro, tem como única restrição o princípio da contabilidade no balanço de pagamentos, fato que, não garante que o padrão de endividamento externo é sustentável em longo prazo. Nesse sentido, Moreno-Brid (1998-99) aperfeiçoa o modelo de crescimento com restrição no balanço de pagamentos levando em consideração a trajetória da acumulação da dívida externa. A solução encontrada foi incorporar uma restrição simples da evolução de certos agregados macroeconômicos que poderiam manter constante a relação entre o déficit em transações correntes/PIB.

Various elements support the convenience of adopting this specification of long-term equilibrium of the balance of payments. It can be easily proved that, in the context of the Model (balance-of-payments constraints), this specification implies that the equilibrium growth path of the economy is consistent too with a constant long-term ratio of external debt to domestic income (Moreno-Brid, 1998/99, p. 286).

Recentemente, a extensão de Araujo e Lima (2007) considera a importância da mudança estrutural para o crescimento de longo prazo, que se refere a variações na composição setorial de um sistema econômico, devido à existência de diferentes produtividades e de mudança da demanda particular a cada um dos setores. Desse ponto de vista, estes fatores desempenham 
um papel motor de todo o sistema econômico, pois ele se traduz em crescimento econômico, sem precisar de uma aceleração do crescimento da renda mundial.

O modelo desenvolvido por Araujo e Lima (2007) permite que economias cresçam a taxas maiores e seguras do ponto de vista da restrição externa, ainda que o crescimento econômico mundial não se tenha ampliado. Tal possibilidade é apreendida no modelo por meio de alterações no padrão comercial do país, refletido nos coeficientes de participação relativa dos setores nas pautas de importação e exportação.

Nesse arcabouço teórico as elasticidades renda são ponderadas pela participação do setor no volume de exportação e importação. Dessa maneira, uma alteração na composição das exportações e importações teria importante papel não somente no processo de crescimento da economia, mas também na capacidade de promover uma mudança estrutural (Araujo; Lima, 2007) Do modelo, conclui-se que a taxa de crescimento da renda per capita da economia brasileira é diretamente proporcional ao crescimento das exportações e inversamente proporcional ao crescimento das importações. Portanto, o perfil da pauta comercial e da estrutura produtiva da economia definirá a razão das elasticidades expostas na equação e, consequentemente, a taxa de crescimento da economia:

$$
g_{y}=\frac{\sum_{i=1}^{n-1} \xi a_{n i} a_{i \widehat{n}} \varepsilon_{i}}{\sum_{i=1}^{n-1} a_{n i} a_{\hat{\imath} n} \pi_{i}} g_{z}
$$

onde: $a_{n i}$ são os coeficientes de produção dos $i$ bens de consumo, $a_{i \hat{n}}$ representa o coeficiente de demanda externa dos $j=1, \ldots, J$ parceiros comerciais pelo bem $i, \operatorname{com} i=1, \ldots, n-1$. Já $a_{\hat{\imath} n}$ representa o coeficiente de demanda doméstica pelo bem $i$ produzido externamente. $\varepsilon_{i}$ é a elasticidade-renda das exportações, $\pi_{i}$ é a elasticidade-renda das importações e $\mathrm{g}_{z}$ é a taxa de crescimento da renda mundial.

Com efeito, Araujo e Lima (2007) defendem a tese de que a superação da restrição externa passa pela modificação da estrutura produtiva, que é possível por duas vias: 1) por uma alteração da produtividade através de políticas de incentivo à tecnologia e inovação; 2) pela taxa de câmbio real, que afeta a estrutura de custos relativos, podendo baratear a produção, ao passo que reduz o salário real, conferindo vantagem competitiva via preço. Contudo, os autores não introduziram explicitamente nem a taxa de câmbio real nem a produtividade no modelo multissetorial. Assim, pretende-se contribuir para o debate, incluindo estas variáveis no modelo.

\section{O comportamento da demanda setorial (Pasinetti, 1993) e sua implicação sobre o progresso tecnológico - a lei de Kaldor-Verdoorn (1966-67)}

De acordo com Pasinetti (1993), a demanda setorial per capita varia exponencialmente, assumindo a seguinte forma:

$$
a_{\text {in }}=a_{\text {in }}(0) e^{r_{i} t}
$$


onde: $a_{i n}$ é a demanda do bem do setor $i ., a_{i n(0)}$ é a demanda inicial do bem do setor i, $r_{i}$ é a taxa de variação da demanda setorial.

A proposta, ainda que interessante, pode ser aperfeiçoada, já que esta concepção é aparentemente um caso particular de um caso mais geral. Ademais, o próprio Pasinetti reconhece que há um nível de saturação para cada mercadoria em função da Lei de Engel. Com efeito, a função que melhor descreve o comportamento dos coeficientes de demanda setoriais é uma logística, tal como proposta por Verhulst (1838):

$$
a_{\text {in }}=\frac{a_{\text {in }}(0) e^{r_{i} t}}{1+a_{\text {in }}(0)\left(e^{r_{i} t}-1\right) / a_{\text {in }}^{*}}(4)
$$

onde $r_{i}$ é a taxa máxima de variação da demanda setorial (mesma taxa do modelo de Pasinetti) e $a_{i n}^{*}$ é o nível de saturação da demanda pelo bem $i$.

Da equação (4), nota-se que o nível de $a_{i n}$ aumenta progressivamente de $a_{\text {in }}(0)$, no tempo $t=0$, até o limite $a_{i n}^{*}$, que é apenas alcançado quando $t \rightarrow \infty$. Assim, têm-se três possíveis cenários para a variação da demanda setorial, a saber:

$$
\left\{\begin{array}{c}
\text { Se } a_{i n} \rightarrow a_{i n}^{*}=>\dot{a}_{i n} \rightarrow 0 \\
\text { Se } 0<a_{i n}<a_{i n}^{*}=>0<\dot{a}_{i n}<r_{i} \\
\text { Se } a_{i n} \rightarrow 0=>\dot{a}_{i n} \rightarrow r_{i}
\end{array}\right.
$$

A equação (5) mostra que, se o nível de demanda setorial de um país estiver próximo ao nível de saturação, então a taxa de crescimento da demanda será pequena. Destarte, quanto mais próximo do nível de saturação estiver o setor, em termos de demanda, menor será a taxa de crescimento da demanda deste setor. Analogamente, se o nível de demanda setorial for nulo (ou próximo disso), a taxa de crescimento da demanda deste setor será a proposta por Pasinetti (1993), isto é, exponencial. Assim, à medida que a demanda pelo bem $i$ se expande, aumenta o consumo total desta mercadoria, que se aproxima do seu nível de saturação. Portanto, caso a demanda se aproxime do nível de saturação, então a taxa de variação diminuirá, ou seja, $\lim _{a_{i n} \rightarrow a_{i n}^{*}} \dot{a}_{i n}=0$, a menos que o nível de saturação da demanda setorial aumente $\left[\dot{a}_{i n}^{*}>0\right]$.

Do exposto, pode-se afirmar que a taxa de variação da demanda setorial terá seguinte forma:

$$
\frac{\dot{a}_{i n}}{a_{i n}}=r_{i}\left(1-\frac{a_{i n}}{a_{i n}^{*}}\right)(6)
$$

De acordo com a Lei de Kaldor-Verdoorn, a taxa de crescimento da produtividade setorial depende da taxa de crescimento da produção setorial que, dada a hipótese do gasto total, é equivalente a demanda setorial:

$$
\frac{\dot{a}_{n i}}{a_{n i}}=\theta_{i}+\alpha_{i} \frac{\dot{a}_{i n}}{a_{i n}}(7)
$$


onde $\theta_{i}$ é o coeficiente de Verdoorn e $\frac{\dot{a}_{i n}}{a_{i n}}$ denota a taxa de crescimento da quantidade produzida do bem $i$.

Para explicar a produtividade ou progresso tecnológico como a razão do produto do setor $i$ sobre a força de trabalho alocado em $i$, deve-se recorrer a Kaldor ${ }^{1}$. $\mathrm{O}$ autor afirma em uma série de generalizações empíricas, que: i) existe uma forte relação da produção manufatureira e o crescimento do PIB real; ii) há uma relação positiva entre a taxa de crescimento da produtividade no setor manufatureiro e o crescimento da produção manufatureira, como resultado de rendimentos crescentes; e iii) quanto mais rápido for o crescimento da produção do setor manufatureiro, maior a taxa de transferência de trabalhadores dos demais setores para o setor manufatureiro, consequentemente, maior a produtividade e o crescimento do país.

A contribuição é compatível com a teoria neoschumpeteriana, na qual os mecanismos de learning by doing, dentre outros, tornam-se importantes fontes de catching up e de redução do hiato tecnológico entre os países. Nessa perspectiva teórica, a difusão tecnológica é uma função crescente da distância do nível total de apropriação do conhecimento de um país em relação ao país que está na fronteira tecnológica. Assim, o desenvolvimento tecnológico de um país pode ser obtido por meio de atividades imitativas, mas este gera resultados inferiores se comparado àqueles países que desenvolvem atividades inovativas internamente. $\mathrm{O}$ nível de atividades inovativas pode ser mensurado por medidas de esforço, gasto em P\&D ou por variáveis de resultados, como números de patentes e índices de inovação.

Destarte, substituindo (6) em (7), tem-se a taxa de crescimento do progresso técnico setorial, dado por:

$$
\frac{\dot{a}_{n i}}{a_{n i}}=\theta_{i}+\alpha_{i}\left[r_{i}\left(1-\frac{a_{i n}}{a_{i n}^{*}}\right)\right](8)
$$

Onde $\theta_{i}$ representa a parte do progresso técnico setorial que não depende do nível de demanda, $a_{i} r_{i}$ representa a taxa máxima de crescimento do progresso técnico setorial e $\frac{a_{i n}}{a_{i n}^{*}}$ representa o nível de saturação da demanda per capita setorial.

Logo, a taxa de variação da demanda setorial no tempo será:

$$
\dot{a}_{n i}=a_{n i} \theta_{i}+a_{n i} \alpha_{i} r_{i}\left(1-\frac{a_{i n}}{a_{i n}^{*}}\right)(9)
$$

Na Figura 1, tem-se a representação do comportamento do progresso tecnológico de cada um dos países estilizados, considerando $\theta_{i}=0$. Por ser endógena e explicada pelo comportamento da demanda, ela possui um formato funcional similar ao da demanda setorial. A definição se baseia na distância com relação à fronteira tecnológica. A primeira curva do progresso tecnológico setorial representa os países desenvolvidos, que atingem a fronteira tecnológica mais rapidamente. Esses países são exatamente aqueles da fronteira tecnológica na

(1) Thirlwall (1983). 
maioria dos setores da atividade econômica. A segunda curva referente ao progresso tecnológico setorial dos países emergentes indica que a evolução é relativamente mais lenta que aquela observada nos países ricos, porém alcança o estágio de desenvolvimento tecnológico com mais velocidade que os países subdesenvolvidos, representados pela terceira curva. Esses países menos desenvolvidos, por sua vez, se beneficiam do progresso tecnológico de duas maneiras, através da inovação e da difusão tecnológica, sendo que esta última tem uma importância relativamente maior quanto menos desenvolvido o país.

Dessa forma, o critério utilizado para distinguir a natureza dos países (desenvolvidos, emergentes e subdesenvolvidos) está centrado na definição da taxa de crescimento do progresso técnico setorial, $r_{i}$. Assim, a maior taxa de progresso técnico corresponde aos países em desenvolvimento, seguido pelos países emergentes e, por último com menor taxa, estão os países subdesenvolvidos. A intuição por detrás disso é que a fronteira tecnológica é exógena, até mesmo para os países desenvolvidos. Nesse caso, as principais diferenças tecnológicas entre os países são a velocidade com que os setores de cada país atingem a fronteira e, fixado um instante $\mathrm{t}$, o nível tecnológico disponível em cada país. Isso significa que os países desenvolvidos atingem a fronteira em menos tempo que os demais grupos de países, sendo o grupo dos subdesenvolvidos o último a alcançá-la. Além disso, fixado um ano qualquer, o patamar disponível de tecnologia nos países desenvolvidos é superior àquele disponível nas outras duas categorias de países. Isso, como será visto adiante, faz uma grande diferença no valor da taxa de crescimento econômico.

Figura 1

Evolução da Produtividade Setorial por Categoria de Países

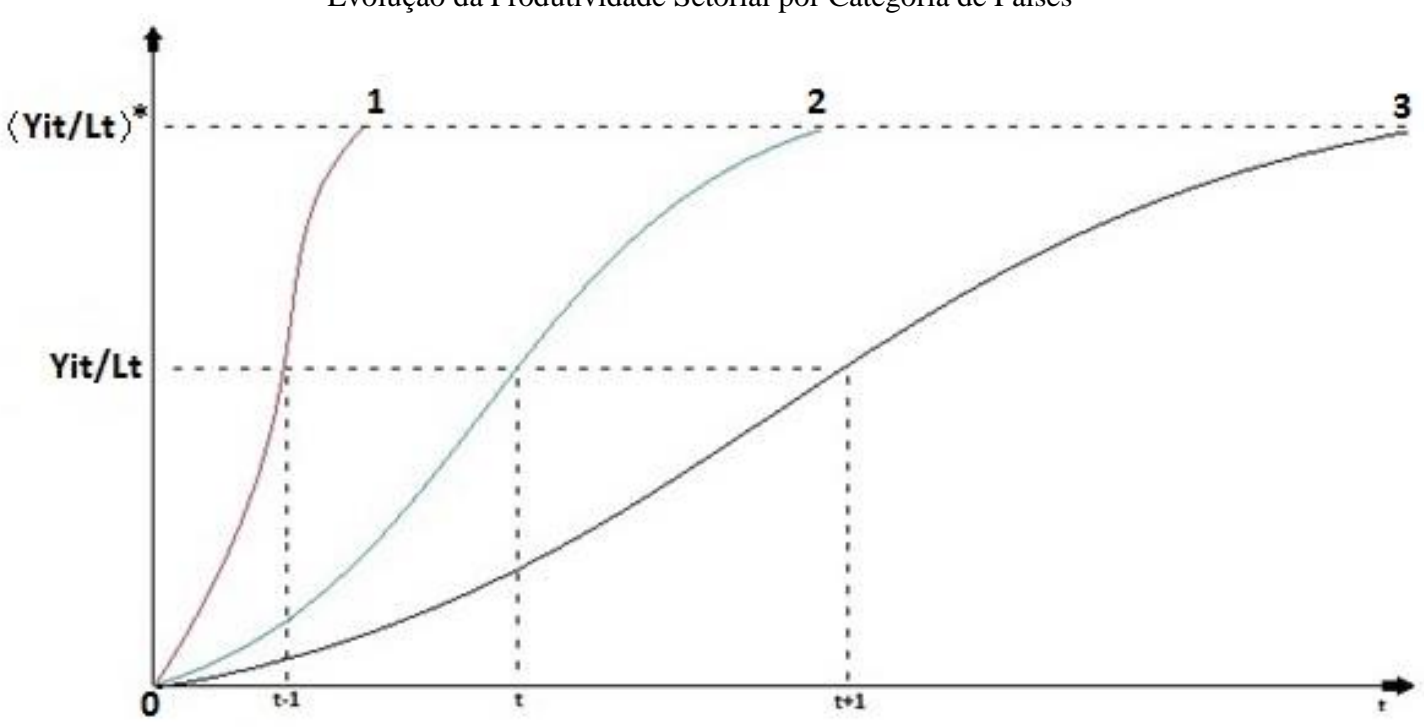

1 - Países Desenvolvidos 2 - Países Emergentes 3 -Países Subdesenvolvidos

Fonte: Elaboração Própria. 
Causação cumulativa, progresso tecnológico endógeno e taxa de câmbio real em uma abordagem dinâmica...

Em suma, no presente artigo, apresenta-se o comportamento da taxa de crescimento da demanda setorial como uma função logística, diferentemente da proposta originalmente por Pasinetti, conferindo um tratamento mais realista ao modelo. Assim, ainda que possa assumir valores negativos ou positivos, a taxa de crescimento da demanda setorial tenderá a se tornar nula no ponto onde o nível de demanda setorial for compatível com a Lei de Engel.

\section{O Modelo Multissetorial de Causação Cumulativa com Taxa de Câmbio Real e Progresso Tecnológico Endógeno}

Neste tópico será apresentada uma versão alternativa do modelo de Araujo e Lima, (2007), considerando também a causação cumulativa, o progresso tecnológico endógeno e a taxa de câmbio real. Dito isto, a condição de pleno emprego é dada por:

$$
\sum_{i=1}^{n-1}\left(a_{i n}+\xi a_{i \hat{n}}\right) a_{n i}=1
$$

Onde $a_{i n}$ e $a_{i \hat{n}}$ representam o coeficiente de demanda pelo bem $i$, com $i=1, \ldots, n-1$. O coeficiente de produção doméstica é dado por $a_{n i}$. O setor que produz mão-de-obra (famílias) externa são denotados por $\hat{n}$ e o tamanho da população em cada um dos países é relacionado através do coeficiente de proporcionalidade $\xi$.

O modelo Pasinettiano assume a existência de pleno emprego apenas no período inicial ( $t=0$. O que o modelo retrata é a situação oposta quando $t>0$, ou seja, desemprego da mãode-obra resultando da interação de progresso tecnológico e variação da demanda particular a cada setor. Isso pode ser depreendido a partir da seguinte afirmação: "Expression (10.5) - by representing at this stage of our analysis the macroeconomic condition for equilibrium growth - warns us that maintaing full employment over time is truly a complex problem" (Pasinetti, 1993, p. 50).

Posta a condição de pleno emprego, cabe apresentar a condição de gasto total:

$$
\sum_{i=1}^{n-1}\left(a_{i n}+a_{\hat{\imath} n}\right) a_{n i}=1(11)
$$

Onde $a_{\hat{\imath} n}$ representa a demanda per capita externa pelo setor iproduzido internamente. A terceira e última condição imposta ao modelo é a de equilíbrio na balança comercial por setor e por parceiro comercial, possuindo a seguinte expressão:

$$
\sum_{i=1}^{n-1}\left(\xi a_{i \hat{n}}-a_{\hat{\imath} n}\right) a_{n i}=0(12)
$$

Diante do exposto, uma importante propriedade deste modelo é que o equilíbrio da balança comercial não pode ser escrito em termos de preço como é usual, mas em termos de coeficiente de trabalho. Portanto, esta condição requer que a quantidade de exportação expressa em termos de quantidades de trabalho do país doméstico seja igual às importações expressas em quantidade de trabalho do país doméstico. A solução deste sistema para quantidades físicas pode ser expressa como:

$$
X_{i}=\left(a_{i n}+\xi a_{i \hat{n}}\right) X_{n}(13)
$$


Assim, a quantidade física de cada bem comercializável que for produzido domesticamente será determinada pela soma da demanda doméstica e pela demanda estrangeira. Com $P_{i t}$ sendo o preço doméstico do bem $i$ no instante corrente e $W$ a taxa (constante) de salário, a solução dos preços pode ser expressa por:

$$
p_{i}=a_{n i} w(14)
$$

Esta solução mostra que a quantidade relativa de trabalho corporificada continua a regular o preço relativo dos bens dentro dos limites de cada país. Diante disso, cabe apresentar as funções exportação e importação. Destarte, a função exportação possui a seguinte forma:

$$
x_{i \hat{n}}=\left\{\begin{array}{lcl}
\left(\frac{P_{i}}{E P_{\hat{\imath}}}\right)^{\eta_{i}} Z^{\varepsilon_{i}} & \text { se } & E P_{\hat{\imath}} \geq P_{i} \\
0 & \text { se } & E P_{\hat{\imath}}<P_{i}
\end{array}\right.
$$

Onde $x_{i \hat{n}}$ representa as exportações do bem $i$ ao parceiro comercial, $\eta_{i}$ é a elasticidade preço da demanda para exportação do bem $i$, com $\eta_{i}<0$, enquanto $\varepsilon_{i}$ é a elasticidade-renda da demanda por exportações por bens produzidos no setor $i$ e $Z$ é a renda do parceiro.

Dividindo ambos os lados de (15) pela população do parceiro, dado $\operatorname{por} X_{\hat{n}}$, tem-se o coeficiente per capita da demanda do parceiro comercial pelo bem $i$ :

$$
a_{i \hat{n}}=\left\{\begin{array}{lll}
\left(\frac{P_{i}}{E P_{\hat{\imath}}}\right)^{\eta_{i}} \frac{Z^{\varepsilon_{i}}}{\left(X_{\hat{n}}\right)^{\varepsilon_{i}}}\left(X_{\hat{n}}\right)^{\varepsilon_{i}-1} & \text { se } & E P_{\hat{\imath}} \geq P_{i} \\
0 & \text { se } & E P_{\hat{\imath}}<P_{i}
\end{array}\right.
$$

Além disto, se o parceiro comercial não possuir vantagem comparativa em produzir o bem $i$, a importação doméstica per capita do bem $i$ produzido por $j$ será zero, isto é, $a_{\hat{\imath} n}=0$. Mas se $P_{i}>E P_{\hat{\imath}}$ então é necessário considerar os coeficientes de demanda de importação, sendo dado por uma função padrão demanda doméstica por importação, que possui a seguinte forma funcional:

$$
x_{\hat{\imath} n}=\left\{\begin{array}{lll}
\left(\frac{E P_{\hat{\imath}}}{P_{i}}\right)^{\Psi_{i}} Y^{\pi_{i}} & \text { se } & P_{i} \geq E P_{\hat{\imath}} \\
0 & \text { se } & P_{i}<E P_{\hat{\imath}}
\end{array}\right.
$$

Onde $\Psi_{i}$ é a elasticidade preço da demanda por importações pelo bem $i$ produzido pelo parceiro comercial, com $\Psi_{i}<0$ e $\pi_{i}$ é a elasticidade-renda da demanda doméstica do setor $i$ por importações produzidas em no setor $i$ do parceiro comercial e $Y$ é a renda real doméstica.

Dividindo ambos os lados de (17) pela população doméstica no período corrente, encontra-se o coeficiente de importação per capita do bem $i$ :

$$
a_{\hat{\imath} n}=\left\{\begin{array}{lcc}
\left(\frac{E P_{\hat{\imath}}}{P_{i}}\right)^{\Psi_{i}} y^{\pi_{i}}\left(X_{n}\right)^{\pi_{i}-1} & \text { se } & P_{i} \geq E P_{\hat{\imath}} \\
0 & \text { se } & P_{i}<E P_{\hat{\imath}}
\end{array}\right.
$$

No caso de $E P_{\hat{\imath}} \geq P_{i}$ pode-se aplicar o logaritmo natural em ambos os lados de (19) e diferenciar em relação ao tempo $t$. Adotando a seguinte convenção: $\frac{\dot{P}_{i}}{P_{i}}=p_{i}, \frac{\dot{P}_{\hat{\imath}}}{P_{\hat{\imath}}}=p_{\hat{\imath}}, \frac{\dot{E}}{E}=e$, 
Causação cumulativa, progresso tecnológico endógeno e taxa de câmbio real em uma abordagem dinâmica...

$\frac{\dot{y}}{y}=\sigma_{y}, \frac{\dot{z}}{z}=\sigma_{z}, \frac{\dot{x}_{n}}{X_{n}}=n, \frac{\dot{X}_{\widehat{n}}}{X_{\widehat{n}}}=\hat{n}$, este procedimento proporciona a seguinte taxa de crescimento per capita da demanda por exportações do bem $i$. Passando o log e derivando (16) em relação ao tempo, tem-se:

$$
\frac{\dot{a}_{i \hat{n}}}{a_{i \hat{n}}}=\left\{\begin{array}{lll}
\eta_{i}\left(p_{i}-p_{\hat{\imath}}-e\right)+\varepsilon_{i} \sigma_{z}+\left(\varepsilon_{i}-1\right) \hat{n} & \text { se } & E P_{\hat{\imath}} \geq P_{i t} \\
0 & \text { se } & E P_{\hat{\imath}}<P_{i t}
\end{array}\right.
$$

Para efeito de simplificação, adota-se que $n=\hat{n}=0$, portanto:

$$
\dot{a}_{i \hat{n}}=\left\{\begin{array}{lll}
a_{i \hat{n}} \eta_{i}\left(p_{i}-p_{\hat{\imath}}-e\right)+a_{i \hat{n}} \varepsilon_{i} \sigma_{z} & \text { se } & E P_{\hat{\imath}} \geq P_{i t} \\
0 & \text { se } & E P_{\hat{\imath}}<P_{i t}
\end{array}\right.
$$

Adotando o mesmo procedimento para a equação (18) para o caso $P_{i} \geq E P_{\hat{\imath}}$ tem-se:

$$
\frac{\dot{a}_{\hat{\imath} n}}{a_{\hat{\imath} n}} \begin{cases}\Psi_{i}\left(p_{\hat{\imath}}+e-p_{i}\right)+\pi_{i} \sigma_{y}+\left(\pi_{i}-1\right) n & \text { se } P_{i} \geq E P_{\hat{\imath}} \\ 0 & \text { se } \quad P_{i t}<E P_{\hat{\imath}}\end{cases}
$$

E, portanto:

$$
\dot{a}_{\hat{\imath} n}=\left\{\begin{array}{lr}
a_{\hat{\imath} n} \Psi_{i}\left(p_{\hat{\imath}}+e-p_{i}\right)+a_{\hat{\imath} n} \pi_{i} \sigma_{y}+a_{\hat{\imath} n}\left(\pi_{i}-1\right) n & \text { se } \quad P_{i} \geq E P_{\hat{\imath}} \\
0 & \text { se } \quad P_{i t}<E P_{\hat{\imath}}
\end{array}\right.
$$

Como não há crescimento da população, então (21a) pode ser reescrita da seguinte maneira:

$$
\dot{a}_{\hat{\imath} n}=\left\{\begin{array}{r}
a_{\hat{\imath} n} \Psi_{i}\left(p_{\hat{\imath}}+e-p_{i}\right)+a_{\hat{\imath} n} \pi_{i} \sigma_{y} \operatorname{seP} P_{i} \geq E P_{\hat{\imath}} \\
\operatorname{seP} P_{i t}<E P_{\hat{\imath}}
\end{array}\right.
$$

Para que a condição de equilíbrio na balança comercial permaneça válida à medida que o tempo passa, é necessário que a seguinte condição seja satisfeita:

$$
\sum_{i=1}^{n-1}\left(\xi \dot{a}_{i \hat{n}}-\dot{a}_{\hat{\imath} n}\right) a_{n i}+\sum_{i=1}^{n-1}\left(\xi a_{i \hat{n}}-a_{\hat{\imath} n}\right) \dot{a}_{n i}=0(22)
$$

Note que embora $\sum_{i=1}^{n-1}\left(\xi a_{i \hat{n}}-a_{\hat{\imath} n}\right) a_{n i}=0$, não se pode concluir que $\sum_{i=1}^{n-1}\left(\xi a_{i \hat{n}}-\right.$ $\left.a_{\hat{\imath} n}\right) \dot{a}_{n i}=0$, pois $a_{n i} \neq \dot{a}_{n i}$. Destarte, apenas em setores onde não há progresso técnico, ou seja, setores onde a produtividade é constante, que as equações serão idênticas. Assim sendo, considera-se neste caso que possa haver crescimento da produtividade ou progresso técnico em cada setor $i$.

Substituindo (9), (20) e (21b) em (22), bem como fazendo os algebrismos necessários, tem-se:

$$
\begin{gathered}
\sigma_{y}=\frac{\sum_{i=1}^{n-1}\left(\xi a_{i \hat{n}}-a_{\hat{\imath} n}\right) a_{n i} \theta_{i}}{\sum_{i=1}^{n-1} a_{n i} a_{\hat{\imath} n} \pi_{i}}+\frac{\sum_{i=1}^{n-1}\left(\xi a_{i \hat{n}} \eta_{i}+a_{\hat{\imath}} \Psi_{i}\right) a_{n i}}{\sum_{i=1}^{n-1} a_{n i} a_{\hat{n} n} \pi_{i}}\left(p_{i}-p_{\hat{\imath}}-e\right)+\frac{\sum_{i=1}^{n-1} \xi a_{n i} a_{i \hat{n}} \varepsilon_{i}}{\sum_{i=1}^{n-1} a_{n i} a_{\hat{\imath} n} \pi_{i}} \sigma_{z}+ \\
\frac{\sum_{i=1}^{n-1}\left(\xi a_{i \hat{n}}-a_{\hat{\imath}}\right) a_{n i} \alpha_{i} r_{i}}{\sum_{i=1}^{n-1} a_{n i} a_{\hat{\imath} n} \pi_{i}}\left(1-\frac{a_{i n}}{a_{i n}^{*}}\right)
\end{gathered}
$$


Onde $\sigma_{y}$ depende do intercepto, $\frac{\sum_{i=1}^{n-1}\left(\xi a_{i \hat{n}}-a_{\hat{\imath}}\right) a_{n i} \theta_{i}}{\sum_{i=1}^{n-1} a_{n i} a_{\hat{\imath}} \pi_{i}}$, da taxa de crescimento real do câmbio por setor, $\left(p_{i}-p_{\hat{\imath}}-e\right)$, da taxa de crescimento econômico do parceiro comercial, $\sigma_{z}$, e do nível de demanda por cada bem em relação ao nível de saturação, respectivamente.

Por simplicidade, defina: $\Delta_{I n}=\sum_{i=1}^{n-1}\left(\xi a_{i \hat{n}}-a_{\hat{\imath} n}\right) a_{n i} \theta_{i}, \Delta_{\pi}=\sum_{i=1}^{n-1} a_{n i} a_{\hat{\imath} n} \pi_{i}, \Delta_{a_{i n}}=$ $\sum_{i=1}^{n-1}\left(\xi a_{i \hat{n}}-a_{\hat{\imath} n}\right) a_{n i} \alpha_{i} r_{i}, \Delta_{e}=\sum_{i=1}^{n-1}\left(\xi a_{i \hat{n}} \eta_{i}+a_{\hat{\imath} n} \Psi_{i}\right) a_{n i} \mathrm{e} \Delta_{z}=\sum_{i=1}^{n-1} \xi a_{n i} a_{i \hat{n}} \varepsilon_{i}$. Este último representa a elasticidade emprego do produto, que capta a influência de fatores extra preço na competitividade de cada setor $i$ no mercado internacional, tais como o conteúdo tecnológico das exportações líquidas do setor $i$ ao parceiro comercial, o grau de diferenciação das exportações líquidas do setor $i$, o valor adicionado a estes produtos, entre outras coisas, sobre a competitividade externa de um país. Vale a pena ressaltar o parâmetro $\Delta_{\text {In }}$ que representa o nível tecnológico setorial. Caso haja uma onda de inovações tecnológicas - incrementos na produtividade ou no próprio paradigma tecnológico não oriundos da aprendizagem aumentarão o nível mínimo da taxa de crescimento econômico doméstico. Desta forma, o nível tecnológico propicia, em certos aspectos, uma medida para a taxa mínima de crescimento econômico, isto é, numa situação extrema e hipotética, quando $\sigma_{z}=\left(p_{i}-p_{\hat{\imath}}-e\right)=0$ e $\left[1-\left(a_{i n} / a_{i n}^{*}\right)\right]=0$ então:

$$
\sigma_{y}=\frac{\Delta_{I n}}{\Delta_{\pi}}(24)
$$

Neste sentido, há uma importância central no nível tecnológico pois, dentre outras coisas, garante um nível mínimo de crescimento econômico.

Do exposto, pode-se representar a equação final do modelo de maneira simplificada como segue:

$$
\sigma_{y}=\frac{\Delta_{I n}}{\Delta_{\pi}}+\frac{\Delta_{z}}{\Delta_{\pi}} \sigma_{z}+\frac{\Delta_{e}}{\Delta_{\pi}}\left(p_{i}-p_{\hat{\imath}}-e\right)+\frac{\Delta_{a_{i n}}}{\Delta_{\pi}}\left(1-\frac{a_{i n}}{a_{i n}^{*}}\right)
$$

A Lei de Thirlwall, derivada num contexto multissetorial passinettiano, tal como realizada por Araujo e Lima (2007), postula que a taxa de crescimento per capita de um país está diretamente relacionada às elasticidades-renda das exportações setoriais multiplicadas pela taxa de crescimento da economia mundial e inversamente relacionada às elasticidades-renda setoriais das importações $\left[\frac{\Delta_{z}}{\Delta_{\pi}} \sigma_{z}\right]$. Essa intuição permanece nesse modelo com tecnologia.

A equação 25 permite captar os efeitos sobre a taxa de crescimento do câmbio sobre o crescimento de longo prazo $\left[\left(p_{i}-p_{\hat{\imath}}-e\right) \frac{\Delta_{e}}{\Delta_{\pi}}\right]$. Intuitivamente, a equação demonstra ainda que o lado da oferta importa na determinação do crescimento das economias e os investimentos em inovação tecnológica $\left[\frac{\Delta_{I n}}{\Delta_{\pi}}\right]$ são fundamentais, principalmente, para os países que desejam melhorar o seu ritmo de crescimento econômico, permitindo uma redução do hiato do produto. A equação ressalta que os policy makers precisam estar mais atentos às políticas de investimentos no setor de desenvolvimento tecnológico, em particular, às políticas de 
investimento direcionadas aos setores mais dinâmicos no sentido de Thirlwall, que são os setores que apresentam as maiores razões das elasticidades da demanda por exportações e importações. Assim, torna-se importante a identificação desses setores para um direcionamento correto dessas políticas de investimentos setoriais.

Evidentemente, os policy makers dos países menos desenvolvidos devem estimular a imitação ou importação de máquinas e equipamentos para permitir que suas economias cresçam num ritmo maior, já que essas economias estão bem abaixo da fronteira tecnológica,

o que significa que podem se beneficiar da difusão tecnológica $\left[\frac{\Delta_{a_{i n}}}{\Delta_{\pi}}\left(1-\frac{a_{i n}}{a_{i n}^{*}}\right)\right]$. Portanto, os resultados demonstram matematicamente que o crescimento no longo prazo depende da capacidade de inovação e de exploração dos benefícios das novas tecnologias desenvolvidas, assim como da capacidade de imitação, pelos países que estão longe da fronteira tecnológica, por meio da difusão de tecnologia internacional."

Assim, a partir de (25) tem-se condições de verificar quais fatores afetam mais significativamente o crescimento econômico de longo prazo. Desta forma, pode-se definir a melhor estratégia de inserção externa do país, bem como apresentar uma estratégia de crescimento sustentável de longo prazo. Assim, os policy makers terão condições de propor políticas econômicas e industriais que melhorem as relações comerciais e a competitividade da economia, para ajustar a taxa de crescimento econômico de longo prazo e conduzir a economia para um estágio superior de desenvolvimento. Em relação ao progresso tecnológico, tem-se que seu impacto sobre a taxa de crescimento econômico de longo prazo depende, além do componente exógeno, do nível do hiato tecnológico setorial existente entre o país considerado e o nível técnico da fronteira. Sendo assim, quanto mais distante da fronteira técnica setorial, maior o impacto de um incremento técnico setorial sobre o crescimento econômico de longo prazo.

\section{Considerações finais}

O objetivo do trabalho foi desenvolver um modelo multissetorial de causação cumulativa com progresso tecnológico endógeno e taxa de câmbio real. Para tanto, realizou-se uma breve introdução sobre os principais artigos desta abordagem, bem como apresentou-se o debate em torno dessa literatura até a concepção multissetorial desenvolvida por Araujo e Lima (2007).

As principais contribuições do presente artigo consistiram em: i) compatibilizar a forma funcional do comportamento da demanda setorial com a Lei de Engel; ii) endogeneizar a taxa de crescimento do progresso tecnológico pela Lei de Kaldor-Verdoorn; iii) deduzir uma LTMS com taxa de crescimento do câmbio real setorial e progresso tecnológico setorial e iv) fornecer uma ferramenta útil para identificar setores importantes para o crescimento da economia doméstica.

Esta abordagem representa um avanço em relação à literatura recente, pois endogenizar o progresso tecnológico permitiu maior robustez ao modelo, haja vista que comumente esta 
Ricardo Azevedo Araujo, Matheus Silva de Paiva, Guilherme Jonas Costa da Silva

variável é considerada exógena. Diante disso, foi possível levar em consideração os impactos que a demanda setorial gera sobre o progresso tecnológico e, consequentemente, sobre a produção e o crescimento econômico, permitindo assim que sejam identificados quais os setores que mais contribuem para o crescimento de longo prazo da economia doméstica. Isto possibilita uma melhor compreensão da estrutura econômica do país e, portanto, auxilia os policy makers a propor políticas que aperfeiçoem a estratégia de inserção externa do país, a fim de conduzir a economia para um estágio superior de desenvolvimento.

\section{Referências bibliográficas}

ARAUJO, R. A. Assessing the dynamics of terms of trade in a model of cumulative causation and structural change. Germany: University Library of Munich, 2013. (MPRA Paper, n. 46049).

ARAUJO, R. A. Cumulative causation in a structural economic dynamic approach to economic growth and uneven development. MPRA (Munich Personal RePEc Archive), v. 24, p. 1-24, Mar. 2011.

ARAUJO, R. A. New insights from a structural economic dynamic approach to balance of payments constrained growth. MPRA (Munich Personal RePEc Archive), 2011 b.

ARAUJO, R. A.; LIMA, G. T. A structural economics-dynamics approach to balance- of payments constrained growth. Cambridge Journal of Economics, p. 755-774, 2007.

ARAUJO, R.; TEIXEIRA, J. A multi-sector version of the post-Keynesian growth model. In: INTERNATIONAL COLLOQUIUM ON GROWTH, STRUCTURAL CHANGE AND INSTITUTIONS, 8. Proceedings... Ed. by T. Boyland and S. Raghavendra. Galway, Ireland, 2011. p. 25-46.

BOTTA, A. A structuralist north-south model on structural change, economic growth and catching-up. Structural Change and Economic Dynamics, v. 20, p. 61-73, 2009.

BRUTON, H. A reconsideration of import substitution. Journal of Economic Literature, v. 36, p. 903-936, 1998.

CORNWALL, J.; CORNWALL,W. A demand and supply analysis of productivity growth. Structural Change and Economic Dynamics, v. 13, n. 2, p. 203-229, 2002.

D'AGATA, A. Endogenous adaptative dynamics in Pasinetti model of structural change. Metroeconomica, v. 61, n. 2, p. 333-363, 2010.

FIORILLO, F. Rate of growth and sector specialisation coevolution in a Kaldorian export-led growth model. Structural Change and Economic Dynamics, Elsevier, v. 12, n. 1, p. 91-114, Mar. 2001.

KALDOR, N. A model of economic growth. The Economic Journal, v. 67, n. 268, p. 591-624, Dec. 1957. 
Causação cumulativa, progresso tecnológico endógeno e taxa de câmbio real em uma abordagem dinâmica...

KALDOR, N. Causes of the slow rate of growth of the United Kingdom. Cambridge: [s.n.]. 1966.

KALDOR, N. Strategic factors in economic development. Ithaca, NY: New York State School of Industrial and Laubour Relations. Cornell University, 1967.

KALDOR, N. The role of effective demand in the short run and the long run. In: BARRÉRE, A. The foundations of Keynesian analysis. London: MacMillan Press, 1988.

KRUGMAN, P. R. Trigger strategies and price dynamics in equity and foreign exchange markets. National Bureau of Economic Research, Inc.,1987. (NBER Working Papers, n. 2459).

McCOMBIE, J. S. L.; THIRLWALL, A. P. Economic growth and the balance-of-payments constraint. London: The Macmillan Press, 1994.

MORENO-BRID, J. C. Balance-of-paymens constraines economic growth: the case of Mexico. Banca Nazionale del Lavoro Quarterly Review, p. 413-433, 1998.

OCAMPO, A. Beyond reforms: structural dynamics and macroeconomic vulnerability. Stanford University Press, 2005.

PASINETTI, L. L. Structural economic dynamics. Cambridge: Cambrigde University Press, 1993.

PASINETTI, L. L. Structural change and economic growth: a theoretical essay on the dynamics of the wealth of nations. Cambridge University Press, 1983.

REATI, A. Technological revolutions in Pasinetti's model of structural change: productivity and prices. Structural Change and Economic Dynamics, v. 9, n. 2, p. 245-262, Jun. 1998.

ROBERTS M.; SETTERFIELD M. What is endogenous growth theory? In: ARESTIS, P.; BADDELEY, M.; McCOMBIE, J. S. L. (Ed.). Economic growth: new directions in theory and policy. Cheltenham: Edward Elgar, 2007.

SETTERFIELD, M. History versus equilibrium and the theory of economic growth. Cambridge Journal of Economics, Oxford University Press, v. 21, n. 3, p. 365-378, May 1997.

THIRLWALL, A. P. The balance of payments constraint as an explanation of international growth rates differences. Banca Nazionale del Lavoro Quarterly, p. 44-55, 1979.

THIRLWALL, A. P.; HUSSAIN, M. N. The balance of payments constraint, capital flows and growth rate differences between developing countries. Oxford Economic Papers: New Series, 1982.

THIRLWALL. A. P. A plain man's guide to Kaldor's growth laws. Journal of Post Keynesian Economics, v. 5, n. 3, p. 345-358, Spring 1983.

VERHULST, P. F. Notice sur la loi que la population pursuit dans son accroissement. corresp. Math. Phys, v. 10, p.113-121, 1838. 